\title{
BMJ Open SARS-CoV-2 population-based seroprevalence studies in Europe: a scoping review
}

Rebecca Grant (D) , ${ }^{1,2}$ Timothée Dub, ${ }^{3}$ Xanthi Andrianou, ${ }^{4,5}$ Hanna Nohynek, ${ }^{3}$ Annelies Wilder-Smith, ${ }^{6,7}$ Patrizio Pezzotti, ${ }^{4}$ Arnaud Fontanet ${ }^{1,8}$
To cite: Grant R, Dub T, Andrianou $\mathrm{X}$, et al. SARSCoV-2 population-based seroprevalence studies in Europe: a scoping review. BMJ Open 2021;11:e045425. doi:10.1136/ bmjopen-2020-045425

- Prepublication history and supplemental material for this paper is available online. To view these files, please visit the journal online (http://dx.doi. org/10.1136/bmjopen-2020045425).

Received 30 September 2020 Revised 09 February 2021 Accepted 25 February 2021
Check for updates

(C) Author(s) (or their employer(s)) 2021. Re-use permitted under CC BY-NC. No commercial re-use. See rights and permissions. Published by BMJ.

For numbered affiliations see end of article.

Correspondence to Dr Arnaud Fontanet; fontanet@pasteur.fr

\section{ABSTRACT}

Objectives We aimed to review SARS-CoV-2

seroprevalence studies conducted in Europe to understand how they may be used to inform ongoing control strategies for COVID-19.

Design Scoping review of peer-reviewed publications and manuscripts on preprint servers from January 2020 to 15 September 2020.

Primary measure Seroprevalence estimate (and lower and upper Cls). For studies conducted across a country or territory, we used the seroprevalence estimate and the upper and lower Cls and compared them to the total number of reported infections to calculate the ratio of reported to expected infections.

Results We identified 23 population-based seroprevalence studies conducted in Europe. Among 12 general population studies, seroprevalence ranged from $0.42 \%$ among residual clinical samples in Greece to $13.6 \%$ in an area of high transmission in Gangelt, Germany. Of the eight studies in blood donors, seroprevalence ranged from $0.91 \%$ in North-Western Germany to $23.3 \%$ in a high-transmission area in Lombardy region, Italy. In three studies which recruited individuals through employment, seroprevalence ranged from $0.5 \%$ among factory workers in Frankfurt, Germany, to $10.2 \%$ among university employees in Milan, Italy. In comparison to nationally reported cases, the extent of infection, as derived from these seroprevalence estimates, is manyfold higher and largely heterogeneous.

Conclusion Exposure to the virus in Europe has not reached a level of infection that would prevent further circulation of the virus. Effective vaccine candidates are urgently required to deliver the level of immunity in the population.

\section{INTRODUCTION}

With the emergence of a novel pathogen, such as SARS-CoV-2 - the virus that causes COVID-19_initial surveillance focuses primarily on those who are hospitalised with severe disease and those who report symptoms. As a result, early estimates of the extent of infection in the population often struggle to account for mild or asymptomatic infections that do not require medical care. This is further exacerbated when availability of molecular tests for diagnosis of acute

\section{Strengths and limitations of this study}

Population-based SARS-CoV-2 seroprevalence studies have now been conducted in Europe.

- We conducted a systematic search of PubMed for peer-reviewed publications and MedRxiv/BioRxiv for manuscripts on preprint servers from January 2020 to 15 September 2020.

- For studies conducted across a country or territory, we used the seroprevalence estimate and the upper and lower Cls and compared them to the number of reported infections to calculate the ratio of reported to expected infections.

infection or capacity for testing is limited. This may have been the case in the initial stages of the first epidemic peak of COVID-19 in many countries across Europe. Therefore, there is an urgent need for seroprevalence studies to enable refined estimates of the extent of infection, particularly when used in population-based serological surveys. ${ }^{12}$

Understanding the extent of infection is important in the current context of the COVID-19 pandemic. Many countries in Europe were severely impacted by the initial epidemic peak in March to June 2020. Healthcare facilities were overwhelmed by the number of patients requiring hospitalisation and/or admission to intensive care unit; as was public health capacity to (1) identify, isolate, test and care for all COVID-19 cases, and (2) trace and quarantine contacts of known COVID-19 cases. As a result, many countries in Europe were forced to implement blunt public health and social measures to break chains of transmission, such as nationwide stay at home orders, and the closing of borders, workplaces and schools. ${ }^{3}$

During this time in Europe, a number of population-based seroprevalence studies have been conducted. As countries have now lifted many of the initial broad-reaching measures, these studies are important to understand 
the extent of infection in the population, and to refine estimates of disease severity and to enable better understanding of population protection against epidemic peaks. Nonetheless, population-based seroprevalence studies are not without caveats. Notably, the selection of participants, and the biases inherent in the selection, as well as the performance of the assays used to measure antibodies may affect the interpretation of the seroprevalence results. ${ }^{4}$ We provide here a scoping review of the population-based seroprevalence studies from Europe available as of 15 September 2020 and a synthesis on how these results may be used to inform ongoing control strategies for COVID-19.

\section{METHODS}

In addition to routine monitoring of population-based seroprevalence studies, we conducted a systematic search of PubMed for peer-reviewed publications and MedRxiv/ BioRxiv for manuscripts on preprint servers from January 2020 to 15 September 2020. The search keywords included the terms COVID-19, SARS-CoV-2 and seroprevalence. The complete search strategy can be found in the online supplemental material.

\section{Inclusion criteria}

We included publications that met all of the following criteria: (1) seroprevalence study conducted in Europe; (2) study population derived from the general population (rather than a healthcare-based population, or a population subject to a specific outbreak investigation); (3) sufficient detail on the type of assay used and the performance (specificity and sensitivity) of the assay for detecting anti-SARS-CoV-2 antibodies reported in the publication, included as a referenced publication, or made publicly available by the manufacturer in the case of a commercially available assay; (4) date of sample collection for serological testing included; (5) estimate of seroprevalence in the population reported as percentage of the study population with anti-SARS-CoV-2 IgG antibodies.

\section{Article screening}

All identified abstracts were screened in duplicate by two reviewers to assess eligibility criteria for inclusion in analysis. A third reviewer resolved discrepancies. The following data were extracted from each study:

Details of the study: authors, year of publication, country, type of publication (publication in peerreviewed journal or manuscript on preprint server).

Methodology: objectives of the study, methods including study population, sample size and methods of recruitment, assay used, sensitivity and specificity of the assay and how these were determined (reported by manufacturer for commercial assays or determined as part of the study), as well as the population used to determine sensitivity and specificity of the assay.
Outcome: study seroprevalence point estimate (and CI, when reported).

While this may lead to an overestimate as to the performance of the assay, for commercially available assays, the most recently reported specificity and sensitivity data as reported by the manufacturer were reported.

\section{Assessment of bias}

The Joanna Briggs Institute checklist for studies reporting prevalence data was used to identify potential biases. Additionally, the qualitative categories defined by Bobrovitz et $a \tilde{l}$ were used to determine the magnitude of the biases into one of four categories: (1) high: limited certainty in prevalence: the true prevalence may be substantially different from the estimated prevalence; (2) moderate: moderate certainty in the prevalence: the true prevalence is likely to be close to the estimate, but there is a possibility that it is substantially different; (3) low: high certainty in the prevalence estimate: true prevalence is likely close to the estimate; and (4) unclear: there was insufficient information to assess risk of bias.

\section{Further COVID-19 epidemiological information}

We extracted epidemic curve and case counts from the European Centre for Disease Prevention and Control (ECDC) on 19 September 2020 (https://www.ecdc. europa.eu/en/publications-data/download-todays-datageographic-distribution-covid-19-cases-worldwide) for countries/territories in which seroprevalence studies included in our analysis were representative of the country/territory. Blood sample collection dates were overlapped on the epidemic curve to assist with the interpretation of the seroprevalence results.

\section{Comparison of case ascertainment}

For the general population studies that were implemented nationwide or across a territory, we used the seroprevalence estimate and the upper and lower CIs and compared them to the number of reported infections 15 days before the end of the blood sample collection period for the seroprevalence study, based on current understanding of anti-SARS-CoV-2 antibody kinetics and the ability to detect these antibodies in the second week of infection. ${ }^{6}$ This allowed us to estimate the number of infections expected based on the seroprevalence estimate and to calculate the ratio of reported to expected infections.

\section{Patient and public involvement}

Our study involved the secondary analysis of data and, as such, there was no direct patient involvement.

\section{RESULTS}

Routine monitoring of literature on SARS-CoV-2 seroprevalence, in addition to a systematic search for publications on PubMed and the MedRxiv/BioRxiv preprint servers identified 315 publications. Of these, 23 met the inclusion 


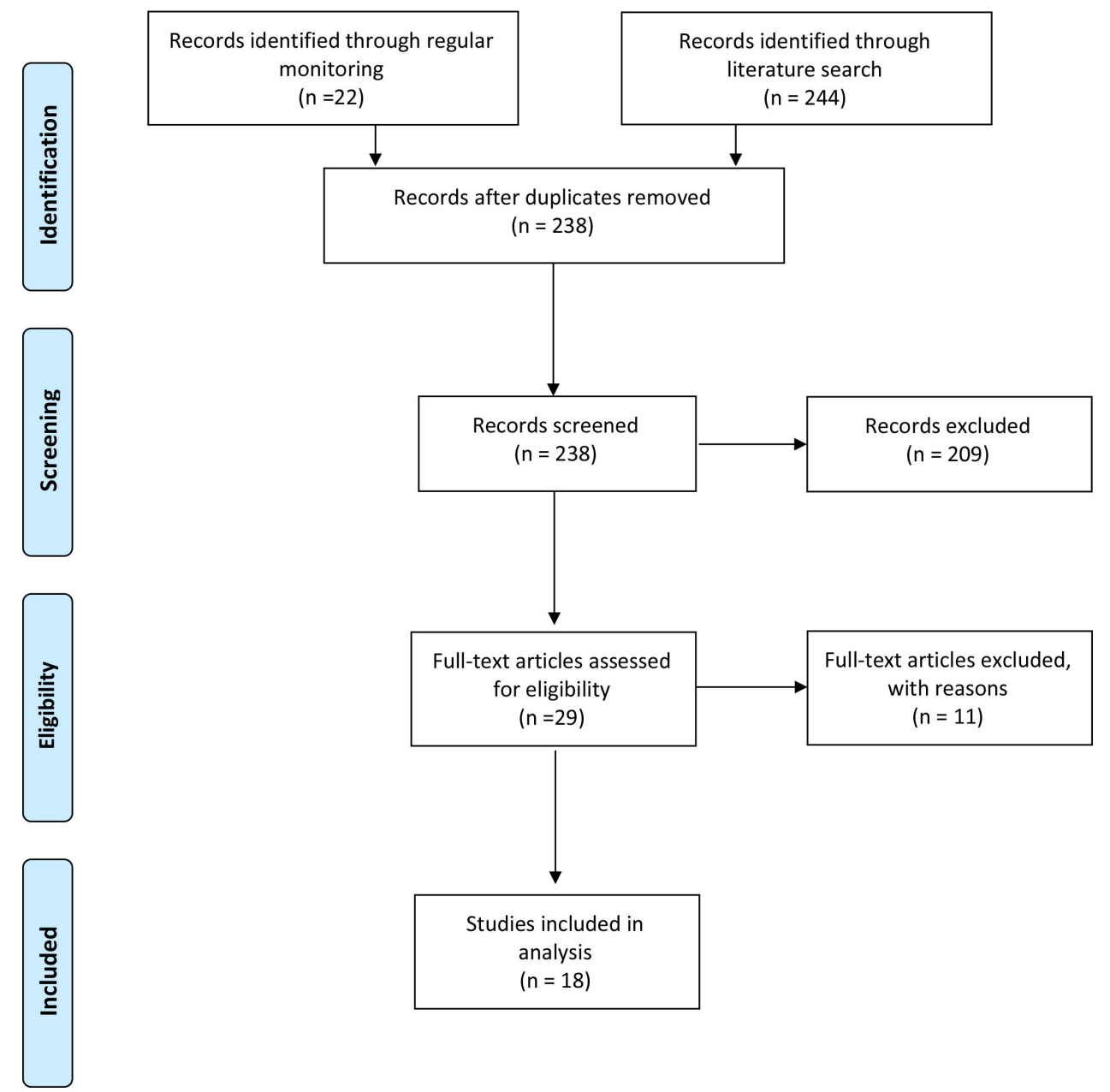

Figure 1 Inclusion of studies in review.

criteria and were included in this review (figure 1). Ten were published in peer-reviewed journals and 13 were manuscripts available on preprint servers (table 1).

Twelve studies ${ }^{7-18}$ used randomly selected samples from the general population, with studies largely conducted through household surveys. A further eight studies ${ }^{19-26}$ were conducted in populations of blood donors, and three additional studies ${ }^{27-29}$ were conducted among individuals who were recruited through employment (table 1).

We did not pool the estimates due to heterogeneity of the populations and in dates of sample collection with respect to SARS-CoV-2 transmission dynamics. Instead, we provide a summary of the seroprevalence estimates based on study population (figure 2). In addition, seven seroprevalence studies were representative of a country/ territory for which epidemic curve and case counts were available from ECDC. ${ }^{7-9} 11121923$ Figure 3 demonstrates that four studies 8111923 involved blood sample collection that included a period of time prior to the first epidemic peak, while three were conducted following the first epidemic peak, as determined by the epidemic curve. ${ }^{7912}$

Figure 3 shows the blood sample collection periods overlayed on the epidemic curves extracted from the European Centre for Disease Prevention and Control.

\section{Population-based seroprevalence studies}

Among the 12 studies conducted in the general population, ${ }^{7-18}$ seroprevalence ranged from $0.42 \%$ among residual clinical samples in Greece ${ }^{7}$ to $13.6 \%$ in an area of high transmission in Gangelt, Germany. ${ }^{15}$ All studies were conducted between March and June 2020, with the studies conducted May to June reflecting the postepidemic peak period in the respective study settings. The largest study was a nationwide cross-sectional study conducted in Spain in which 51958 household members were recruited after the first epidemic peak in the country and found seroprevalence using an immunoassay of $4.6 \%$ (95\% CI 4.3 to 5.0). ${ }^{9}$

Three studies 81011 performed serial sampling of participants. In Geneva, Switzerland, participants from an existing longitudinal cohort study were sampled across 5 consecutive weeks. While the same individuals were not sampled each week, seroprevalence increased: from $4.8 \%$ (95\% CI $2.4 \%$ to $8.0 \%, \mathrm{n}=341)$ in the first week to $10.9 \%$ (95\% CI $7.9 \%$ to $14.4 \%, \mathrm{n}=577$ ) in the third week, before stabilising at $10.8 \%(95 \%$ CI $8.2 \%$ to $13.9 \%, \mathrm{n}=775)$ in the fifth week. ${ }^{10}$

Similarly, in Belgium, residual clinical samples from hospitals and diagnostic laboratories were sampled across five collection periods from the end of March to the start of July. It was estimated that 2.9\% (95\% CI 2.3\% to 3.6\%) 


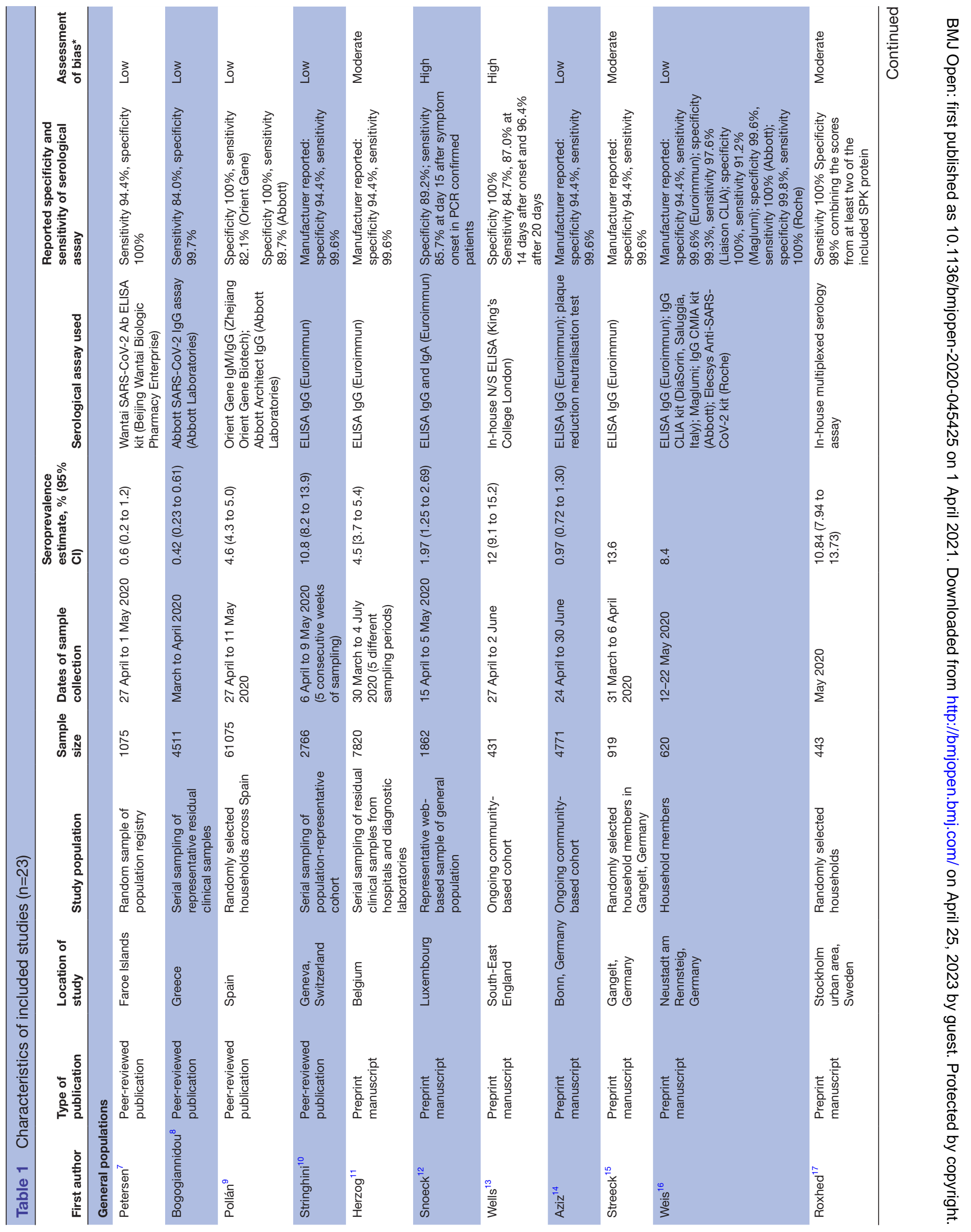




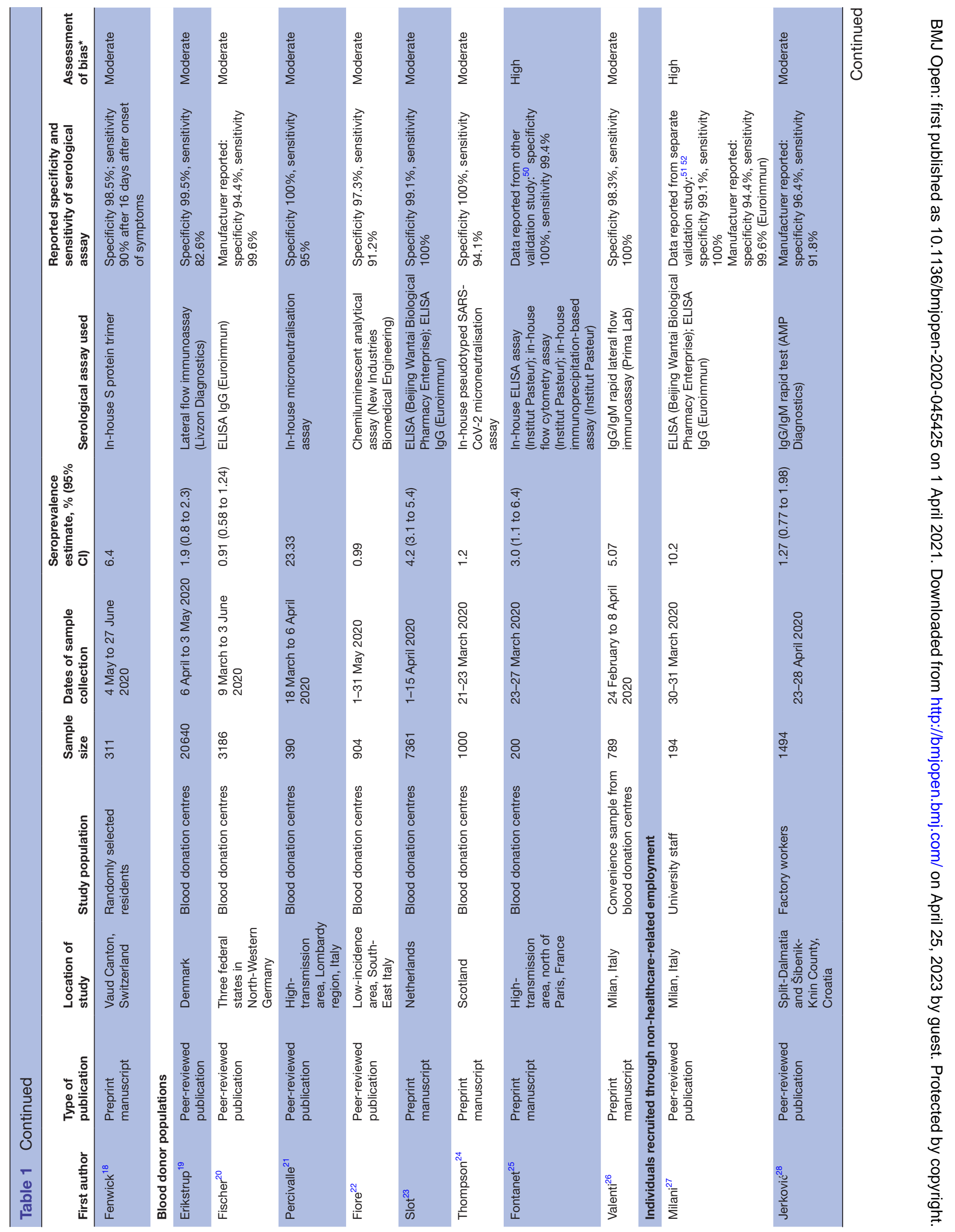




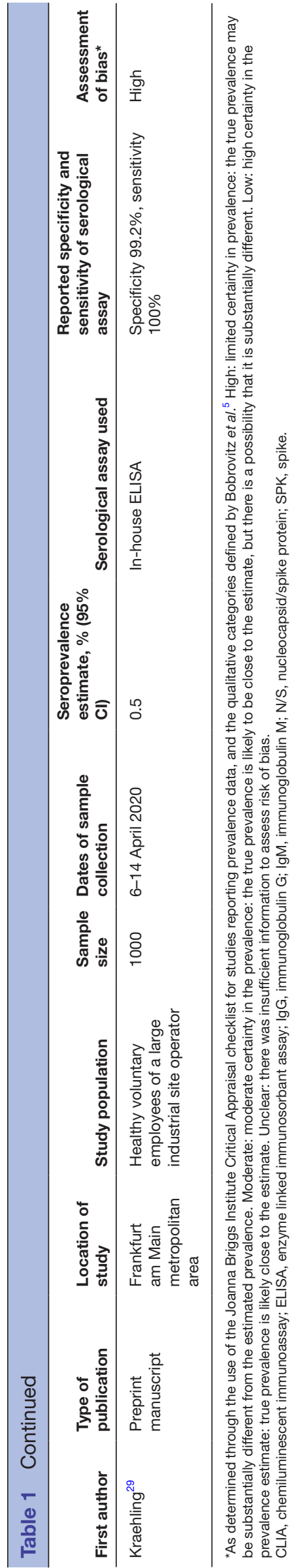

of the Belgian population had detectable antibodies at the end of March, which doubled to $6.0 \%$ (95\% CI 5.1\% to $7.1 \%$ ) 3 weeks later but decreased to $4.5 \%$ (95\% CI $3.70 \%$ to $5.40 \%$ ) in the fifth collection period (29 June to 3 July 2020). ${ }^{10}$ In Greece, residual clinical samples were tested following a geographically stratified sampling plan based on regional units. Seroprevalence increased from $0.24 \%$ (95\% CI $0.03 \%$ to $0.45 \%$ ) in March to $0.42 \%$ (95\% CI $0.23 \%$ to $0.61 \%$ ) in April. ${ }^{8}$

Seven of the 12 studies stratified seroprevalence estimates by age. ${ }^{7-11} 1516$ In the nationwide seroprevalence study conducted in Spain, seroprevalence was found to increase with age and the lowest seroprevalence was found in those aged $0-19$ years, $3.8 \%$ (95\% CI $3.2 \%$ to $4.6 \%){ }^{9}$ In Geneva, Switzerland, seroprevalence was $0.8 \%$ in 5-9 years, compared with $9.6 \%$ in the $10-19$ years and $9.9 \%$ in 20-49 years. ${ }^{10}$ In Belgium and Greece, age-specific seroprevalence from residual clinical samples from hospitals and diagnostic laboratories was found to increase with age. ${ }^{811}$ In Gangelt, Germany, infection rates were found to be lower in the 5-14 years, compared with any other age group. ${ }^{15}$ In Neustadt am Rennsteig, Germany, seroprevalence in children and adolescents was found to be $1.7 \%$, compared with $9.1 \%$ in adults. ${ }^{16}$ In the Faroe Islands, ${ }^{7}$ although estimates are reported by age, only six participants were found to be seropositive so inferences as to age-specific seroprevalence are more difficult.

\section{Seroprevalence studies in blood donors}

Of the eight studies in blood donor populations, ${ }^{19-26}$ seroprevalence ranged from $0.91 \%$ in North-Western Germany to $23.3 \%$ in the area of Lodi province (Lombardy, Italy) where high transmission of COVID-19 was detected from the end of February 2020.

One study in Scotland performed serial sampling on blood samples collected through blood donation centres. All blood samples were negative in mid-March, but rose from end of March. Seroprevalence results were stratified by location across the country, and seroprevalence was found to be heterogeneous by location. In Milan, serial sampling of blood donation samples found the seroprevalence to increase from $2.0 \%$ at the end of February to $5.0 \%$ by mid-March to early April.

While blood donor populations inherently do not include children, several adult blood donor populations were stratified by age. Among 20640 blood donors across Denmark, the youngest (17-29 years: $2.5 \%)$ and oldest (60-69 years: $2.5 \%$ ) blood donors were found to have higher seroprevalence. In South-East Italy, it was the 26-35 years old $(2.0 \%)$ and the $56-65$ years old $(2.0 \%)$ age groups which had the highest seroprevalence.

\section{Seroprevalence studies in employees/individuals recruited} through non-healthcare-related employment

Three studies ${ }^{27-29}$ recruited individuals through employment. University employees without any symptoms in Milan were found to have a seroprevalence of $10.2 \%$; factory workers in two counties in Croatia were found 


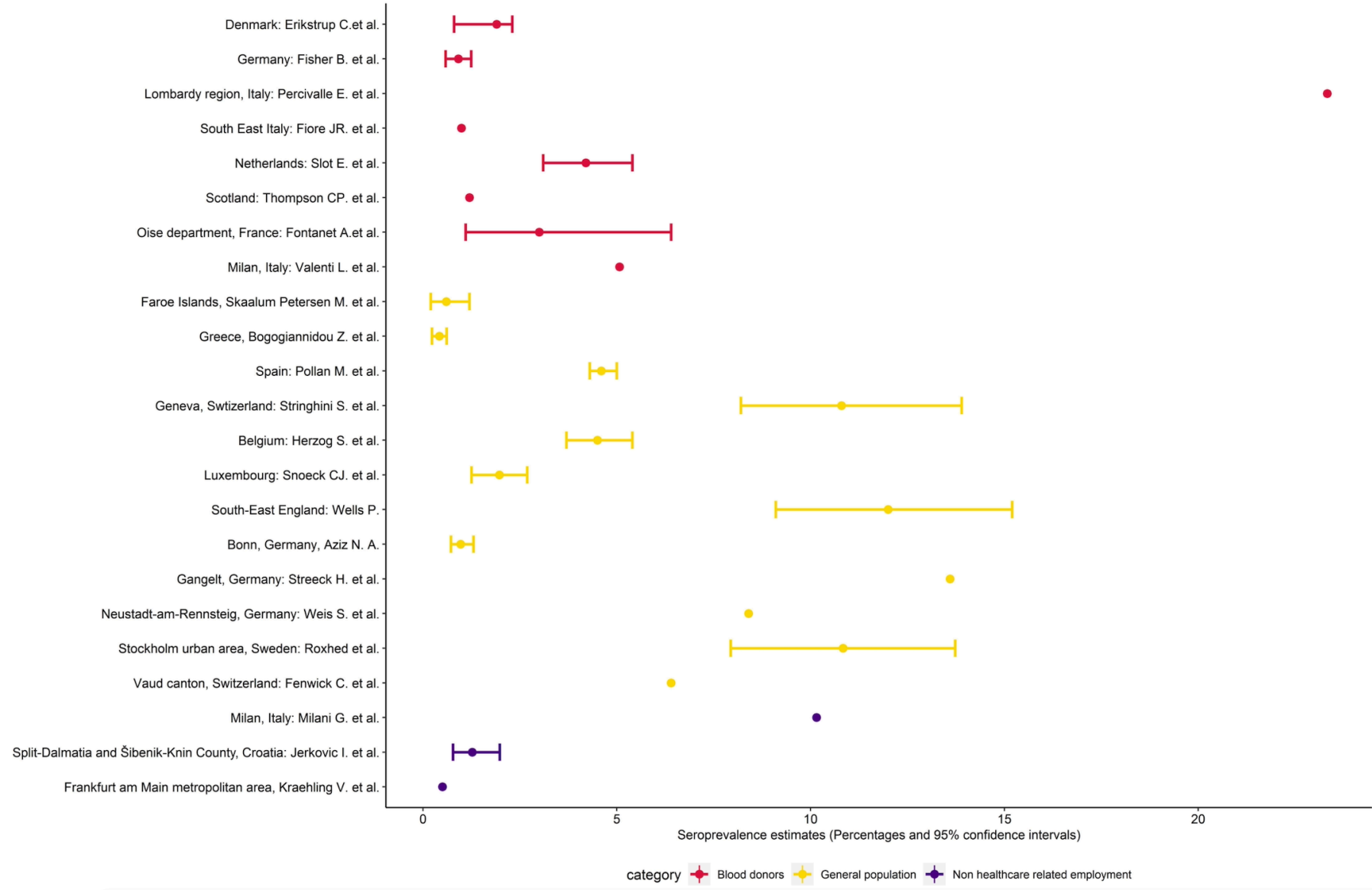

Figure 2 Study seroprevalence (point estimate and Cl, when reported) (For studies that used serial sampling, the most recent seroprevalence estimate was selected).

to have a seroprevalence of $1.3 \%$, while healthy volunteer industrial site operators in the metropolitan area of Frankfurt am Main were found to have a seroprevalence of $0.5 \%$.

\section{Comparison of case underascertainment}

We were able to use the serology-derived estimates of extent of infection in the four general population studies ${ }^{7911} 12$ that were implemented nationwide or across a territory to compare to the total number of reported infections reported 15 days prior to the end of the blood sample collection period by the country/territory. Across the four studies, the ratio of reported to expected number ranged from $10 \%$ to $63 \%$ (table 2 ).

\section{DISCUSSION}

In this scoping review of 23 published seroprevalence studies from Europe, we find heterogeneous results, ranging from $0.42 \%$ among geographically representative residual clinical samples across Greece to $23.3 \%$ in blood donors in an area of high transmission in Lombardy, Italy. The studies in which serial sampling was conducted noted that an increasing fraction of the population has been exposed to the virus. There was no consistency in age stratification so inferences as to differences in seroprevalence by age are difficult to make at this stage.

In comparison to total reported cases of infection, we observed that there was large heterogeneity among countries in the seroprevalence-derived estimates of extent of infection. This likely reflects testing strategies for molecular testing during the first epidemic peak in Europe and the laboratory capacity for diagnosing COVID-19, which in many places was restricted to those with severe disease or those requiring hospitalisation. Understanding testing strategies is an important consideration for analysing and comparing surveillance data, particularly in the COVID-19.

The heterogeneity that we observed in seroprevalence estimates across studies may be explained by several factors. First, the heterogeneity of transmission within Europe and within countries. Across Spain, for example, seroprevalence ranged from $1.2 \%$ to $14.4 \%$, likely reflecting the heterogeneity in transmission intensity across the country. ${ }^{9}$ Second, the study population and the biases inherent in the study design, how the study population has been selected in each study, prevent us from being able to pool seroprevalence estimates. ${ }^{30}$ Eight studies used blood donor populations, which, by definition, select adults without any recent symptoms consistent 

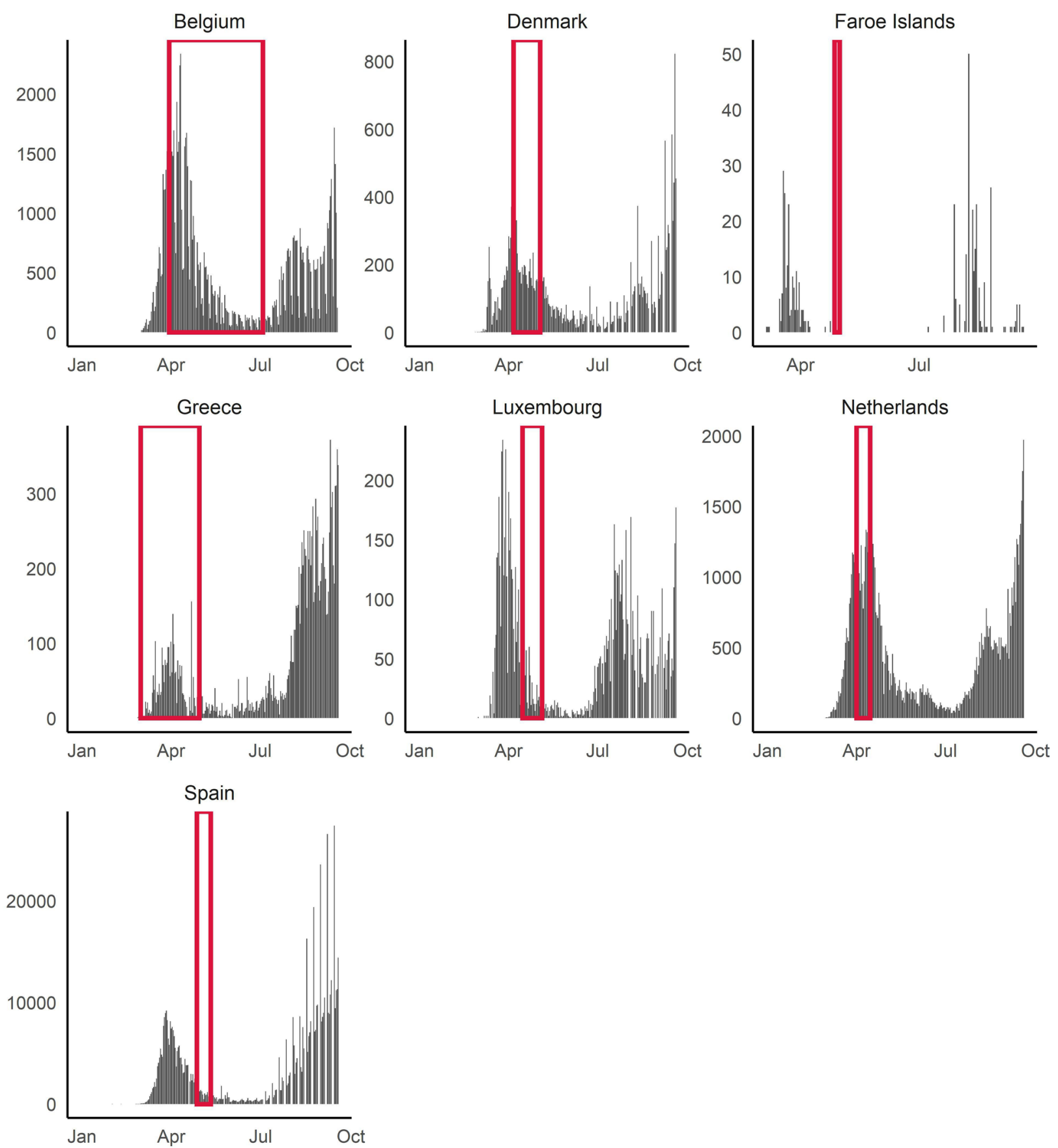

Figure 3 Blood sample collection periods of studies with respect to reported national epidemic curves of reported cases for those studies conducted nationwide/territory-wide $(n=7)$.

with COVID-19. As such, the seroprevalence in blood donors is likely to underestimate the seroprevalence of the general population, particularly in early seroprevalence studies, as is the case in this review. ${ }^{5}$ In addition, this population tends to be healthier than the general population. ${ }^{4}$ The studies among blood donors found seroprevalence to be largely comparable to studies that used household surveys targeting the general population, as shown in table 1 , with the exception of the blood donors in Lombardy. The $23.3 \%$ seroprevalence, measured around the peak of transmission in the Lombardy region, likely reflects the intensity of transmission at that time.
When considering the lag time between infection and measurable antibodies, and the study population, the postpeak seroprevalence in the general population may be in fact substantially higher. That is, those infected at or around the period of most intense transmission (within the 2-3 weeks prior to sample collection) would most likely have had a negative serological test result but would have gone on to seroconvert shortly afterwards.

Further heterogeneity may derive from the type of serological assays and the various performance of the assays. ${ }^{30}$ All assays report high sensitivity as shown in table 1; however, a context of low seroprevalence, as is 


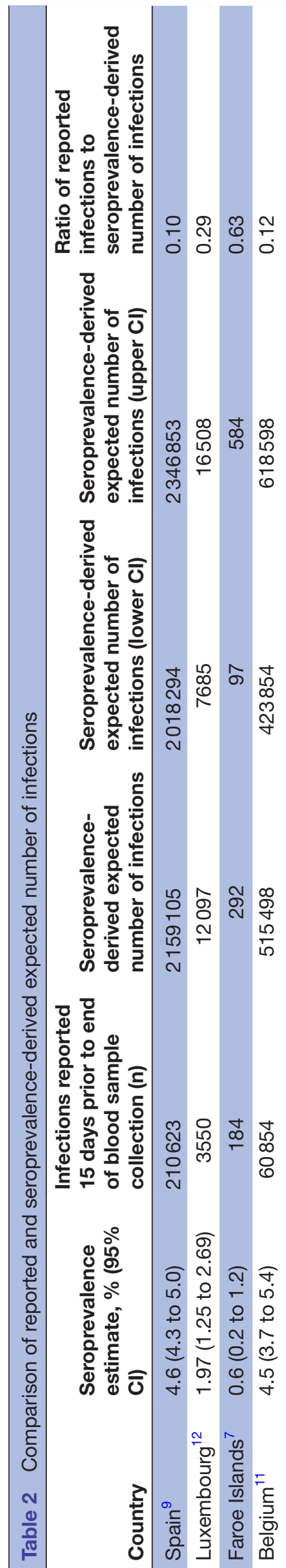

the case for SARS-CoV-2, means low positive predictive value for antibody testing. A number of studies report the validation of the assay used as part of the study, as well as the populations used for this validation. Others report the validation performed as part of other studies, while others simply report the validation data from the manufacturer. A number of studies used the Euroimmun ELISA assay, ${ }^{10-12 ~ 14-16 ~} 202327$ yet the performance of the assay varies in validation studies, likely due to differences in clinical and analytical validity.

A further consideration when interpreting the results of the review is the type of the assay used. Only three of the 23 studies used neutralisation assays, ${ }^{14} 2124$ while the rest used a rapid immunoassay, an ELISA or chemiluminescent immunoassay (CLIA) assay. While the latter detects immunoglobulins specific to SARS-CoV-2, often much quicker and less laboriously than the former, they do not implicitly indicate the strength of an individual's immune response. Neutralisation assays, in contrast, reflect more closely the functional role of anti-SARS-CoV-2 antibodies in the immune response and therefore give a better indication as to protection from further infection. Additional validation studies are required to understand the correlation between antibody titres detected by a rapid immunoassay, ELISA or CLIA, and the neutralising antibody response. This is important-for other coronavirus, individuals who are IgG positive are able to be reinfected, ${ }^{31}{ }^{32}$ and there are now reports of SARS-CoV-2 reinfection. ${ }^{33}$ There are several possible explanations for this, including the implications related to the detection of antibodies versus the detection of neutralising antibodies.

In addition, no longitudinal cohort studies were able to be included in this review. As such, all studies present antibody responses in individuals at one point in time. For the studies that used serial sampling, these were different individuals who were sampled, selected from the same source population each time. We are therefore unable to comment on the duration or persistence of antibodies, nor how this may correlate to ongoing protection. Longitudinal studies that follow the same individuals over time are needed to understand how long antibody, ideally neutralising antibodies, may persist. ${ }^{34}{ }^{85}$

Finally, in addition to the humoral response, the body also mounts a cellular response against infection. Specifically, T-cells recognise and eliminate other cells infected with a virus. By looking only at antibody detection, studies to determine the extent of infection in the population, the study presupposes that everyone who is infected seroconverts, at least to levels that the assay can detect. ${ }^{4}$ The proportion of those who mount a cellular response, but not a detectable antibody response, is currently unclear. ${ }^{36}{ }^{37}$ Further research that combines assessment of the humoral and cellular responses is needed to understand the correlates of protection and to quantify the magnitude of those who may in fact have some protection from infection despite a negative antibody result.

Our findings are limited by the quality of the individual studies. Our assessments showed that many were subject 
to biases. It likely means that the true prevalence may be different from that estimated in the study. For this reason, we did not pool seroprevalence estimates across the region.

Nonetheless, despite this heterogeneity and limitations implicit in the various studies, the picture across Europe after the first epidemic peak of SARS-CoV-2 is clear: exposure to the virus has been insufficient to deliver the level of infection in the population that would be required to prevent further circulation of the virus. The threshold beyond which such herd immunity may be achieved is estimated to be $50 \%-67 \% .{ }^{38}{ }^{39}$ Above this threshold, it is thought that the virus may no longer be able to circulate in the population.

These findings have important policy implications for countries in Europe. While a few experts have recommended that countries seek primarily to achieve herd immunity by allowing the virus to circulate in societies unimpeded, the vast majority of scientists and experts have not recommended this strategy. ${ }^{40}$ This position is based on a number of considerations.

First, such a strategy has and will continue to overwhelm healthcare systems. The devastating effect on the healthcare systems was observed early in the pandemic in countries which were slow to respond to the identification of initial cases. Overwhelmed healthcare systems disrupt the delivery of care to patients with COVID-19 and the delivery of non-COVID-19 health services. ${ }^{41}$ Elective surgeries are delayed, vaccine campaigns are halted and access to healthcare may be difficult.

Further, we now understand that transmission of SARS-CoV-2 is largely concentrated in close-contact settings through large droplets, aerosols and contaminated surfaces. ${ }^{42}$ Targeting these high-risk settings for control measures will create a large reduction in transmission rates, more so than the blunt public health and social measures, and with the advantage of avoiding the adverse economic and societal impacts. Further, contact tracing and epidemiological studies indicate that a small proportion of all people infected likely account for a much larger proportion of onward transmission, ${ }^{43-46}$ although age-specific rates of contacts also likely influence transmission and immunity patterns. ${ }^{47}$

Overall, the results of the initial seroepidemiological studies in Europe indicate the population immunity is below the likely threshold for herd immunity and that measures to (1) identify, isolate, test and care for all COVID-19 cases, and (2) trace and quarantine contacts of known COVID-19 cases will need to be maintained far beyond the emergence of COVID-19 and the initial epidemic peak. ${ }^{48}$ In parallel, efficient roll-out of effective vaccines is needed to deliver the required level of herd immunity in the population.

\section{Author affiliations}

${ }^{1}$ Emerging Diseases Epidemiology Unit, Institut Pasteur, Paris, France

${ }^{2}$ Sorbonne Université, Paris, France
${ }^{3}$ Department of Health Security, Finnish Institute for Health and Welfare, Helsinki, Finland

${ }^{4}$ Department of Infectious Diseases, Italian National Institute of Health (Istituto Superiore di Sanità), Rome, Italy

${ }^{5}$ Cyprus International Institute for Environmental and Public Health, Cyprus University of Technology, Limassol, Cyprus

${ }^{6}$ Department of Disease Control, London School of Hygiene and Tropical Medicine, London, UK

${ }^{7}$ Heidelberg Institute of Global Health, University of Heidelberg, Heidelberg, Germany ${ }^{8}$ PACRI Unit, Conservatoire National des Arts et Métiers, Paris, France

Contributors $A F, R G, T D$ and $X A$ designed the review. RG, TD and $X A$ conducted the literature search and extracted the data from eligible studies. TD, XA and PP performed statistical analyses. RG, TD and XA drafted the first versions of the manuscript. HN, AWS, PP and AF critically revised the first version of the manuscript. All authors reviewed and approved the final version of the manuscript.

Funding The authors have not declared a specific grant for this research from any funding agency in the public, commercial or not-for-profit sectors.

Competing interests None declared.

Patient consent for publication Not required.

Provenance and peer review Not commissioned; externally peer reviewed.

Data availability statement All data relevant to the study are included in the article or uploaded as supplemental information. No additional data available.

Supplemental material This content has been supplied by the author(s). It has not been vetted by BMJ Publishing Group Limited (BMJ) and may not have been peer-reviewed. Any opinions or recommendations discussed are solely those of the author(s) and are not endorsed by BMJ. BMJ disclaims all liability and responsibility arising from any reliance placed on the content. Where the content includes any translated material, BMJ does not warrant the accuracy and reliability of the translations (including but not limited to local regulations, clinical guidelines, terminology, drug names and drug dosages), and is not responsible for any error and/or omissions arising from translation and adaptation or otherwise.

Open access This is an open access article distributed in accordance with the Creative Commons Attribution Non Commercial (CC BY-NC 4.0) license, which permits others to distribute, remix, adapt, build upon this work non-commercially, and license their derivative works on different terms, provided the original work is properly cited, appropriate credit is given, any changes made indicated, and the use is non-commercial. See: http://creativecommons.org/licenses/by-nc/4.0/.

ORCID iD

Rebecca Grant http://orcid.org/0000-0001-8412-8113

\section{REFERENCES}

1 Alter G, Seder R. The power of antibody-based surveillance. N Engl J Med 2020;383:1782-4.

2 Koopmans M, Haagmans B. Assessing the extent of SARS-CoV-2 circulation through serological studies. Nat Med 2020;26:1171-2.

3 Flaxman S, Mishra S, Gandy A, et al. Estimating the effects of non-pharmaceutical interventions on COVID-19 in Europe. Nature 2020;584:257-61.

4 Clapham H, Hay J, Routledge I, et al. Seroepidemiologic study designs for determining SARS-COV-2 transmission and immunity. Emerg Infect Dis 2020;26:1978-86.

5 Bobrovitz N, Arora RK, Yan T. Lessons from a rapid systematic review of early SARS-CoV-2 serosurveys. MedRxiv 2020.

6 Sethuraman N, Jeremiah SS, Ryo A. Interpreting diagnostic tests for SARS-CoV-2. JAMA 2020;323:2249-51.

7 Petersen MS, Strøm M, Christiansen DH. Seroprevalence of SARSCoV-2-specific antibodies, Faroe Islands. Emerg Infect Dis 2020.

8 Bogogiannidou Z, Vontas A, Dadouli K, et al. Repeated leftover serosurvey of SARS-CoV-2 IgG antibodies, Greece, March and April 2020. Euro Surveill 2020;25:2001369.

9 Pollán M, Pérez-Gómez B, Pastor-Barriuso R, et al. Prevalence of SARS-CoV-2 in Spain (ENE-COVID): a nationwide, population-based seroepidemiological study. Lancet 2020;396:535-44.

10 Stringhini S, Wisniak A, Piumatti G, et al. Seroprevalence of antiSARS-CoV-2 IgG antibodies in Geneva, Switzerland (SEROCoVPOP): a population-based study. Lancet 2020;396:313-9.

11 Herzog S, De Bie J, Abrams S. Seroprevalence of IgG antibodies against SARS coronavirus 2 in Belgium: a prospective crosssectional nationwide study of residual samples. MedRxiv 2020. 
12 Snoeck CJ, Vaillant M, Abdelrahman T. Prevalence of SARS-CoV-2 infection in the Luxembourgish population: the CON-VINCE study. MedRxiv 2020.

13 Wells PM, Doores KM, Couvreur S. Estimates of the rate of infection and asymptomatic COVID-19 disease in a population sample from se England. MedRxiv 2020

14 Aziz NA, Corman VM, Antje KC. Seroprevalence and correlates of SARS-CoV-2 neutralizing antibodies: results from a population-based study in Bonn, Germany. MedRxiv 2020.

15 Streeck H, Schulte B, Kuemmerer B. Infection fatality rate of SARSCoV-2 infection in a German community with a super-spreading event. MedRxiv 2020

16 Weis S, Scherag A, Baier M. Seroprevalence of SARS-CoV-2 antibodies in an entirely PCR-sampled and quarantined community after a COVID-19 outbreak - the CoNAN study. MedRxiv 2020.

17 Roxhed N, Bendes A, Dale M. A translational multiplex serology approach to profile the prevalence of anti-SARS-CoV-2 antibodies in home-sampled blood. MedRxiv 2020.

18 Fenwick C, Croxatto A, Coste AT. Changes in SARS-CoV-2 antibody responses impact the estimates of infections in population-based seroprevalence studies. MedRxiv 2020.

19 Erikstrup C, Hother CE, Pedersen OBV, et al. Estimation of SARSCoV-2 infection fatality rate by real-time antibody screening of blood donors. Clin Infect Dis 2020 doi:10.1093/cid/ciaa849

20 Fischer B, Knabbe C, Vollmer T. SARS-CoV-2 IgG seroprevalence in blood donors located in three different federal states, Germany, March to June 2020. Euro Surveill 2020;25:2001285

21 Percivalle E, Cambiè G, Cassaniti I, et al. Prevalence of SARS-CoV-2 specific neutralising antibodies in blood donors from the Lodi red zone in Lombardy, Italy, as at 06 April 2020. Euro Surveill 2020;25.

22 Fiore JR, Centra M, De Carlo A. Results from a survey in healthy blood donors in South eastern Italy indicate that we are far away from herd immunity to SARS-CoV-2. J Med Virol 2020:1-4.

23 Slot E, Hogema BM, Reusken C. Herd immunity is not a realistic exit strategy during a COVID-19 outbreak. Research Square 2020

24 Thompson CP, Grayson NE, Paton RS, et al. Detection of neutralising antibodies to SARS-CoV-2 to determine population exposure in Scottish blood donors between March and may 2020. Euro Surveill 2020;25.

25 Fontanet A, Tondeur L, Madec Y, et al. Cluster of COVID-19 in northern France: a retrospective closed cohort study. SSRN Journal 2020.

26 Valenti L, Bergna A, Pelusi S. SARS-CoV-2 seroprevalence trends in healthy blood donors during the COVID-19 outbreak in Milan. Blood Transfus 2021 doi:10.2450/2021.0324-20

27 Milani GP, Montomoli E, et al, UNICORN Consortium investigators. SARS-CoV-2 infection among asymptomatic homebound subjects in Milan, Italy. Eur J Intern Med 2020;78:161-3.

28 Jerković I, Ljubić T, Bašić Ž, et al. SARS-CoV-2 antibody seroprevalence in industry workers in Split-Dalmatia and ŠibenikKnin County, Croatia. J Occup Environ Med 2021;63:32-7.

29 Kraehling V, Kern M, Halwe S. Epidemiological study to detect active SARS-CoV-2 infections and seropositive persons in a selected cohort of employees in the Frankfurt am main metropolitan area. MedRxiv 2020

30 Kadkhoda K. Letter to the editor: COVID-19: how accurate are seroprevalence studies? Euro Surveill 2020;25:2001374.

31 Padron-Regalado E. Vaccines for SARS-CoV-2: lessons from other coronavirus strains. Infect Dis Ther 2020:1-20.

32 Edridge AWD, Kaczorowska J, Hoste ACR, et al. Seasonal coronavirus protective immunity is short-lasting. Nat Med 2020;26:1691-3.
33 To KK-W, Hung IF-N, Ip JD, et al. COVID-19 re-infection by a phylogenetically distinct SARS-coronavirus-2 strain confirmed by whole genome sequencing. Clin Infect Dis 2020:ciaa1275.

34 Long Q-X, Tang X-J, Shi Q-L, et al. Clinical and immunological assessment of asymptomatic SARS-CoV-2 infections. Nat Med 2020;26:1200-4.

35 Seow J, Graham C, Merrick B. Longitudinal evaluation and decline of antibody responses in SARS-CoV-2 infection. MedRxiv 2020.

36 Wu F, Wang A, Liu M, et al. Neutralizing antibody responses to SARS-CoV-2 in a COVID-19 recovered patient cohort and their implications. SSRN Journal 2020.

37 Sekine T, Perez-Potti A, Rivera-Ballesteros O, et al. Robust T cell immunity in convalescent individuals with asymptomatic or mild COVID-19. Cell 2020;183:158-68.

38 Syal K. COVID-19: herd immunity and convalescent plasma transfer therapy. J Med Virol 2020;92:1380-2.

39 Anderson RM, Heesterbeek $\mathrm{H}$, Klinkenberg D, et al. How will countrybased mitigation measures influence the course of the COVID-19 epidemic? Lancet 2020;395:931-4.

40 Fontanet A, Cauchemez S. COVID-19 herd immunity: where are we? Nat Rev Immunol 2020;20:583-4.

41 Stöß C, Steffani M, Kohlhaw K, et al. The COVID-19 pandemic: impact on surgical departments of non-university hospitals. BMC Surg 2020;20:313

42 Alwan NA, Burgess RA, Ashworth S, et al. Scientific consensus on the COVID-19 pandemic: we need to act now. Lancet 2020;396:e71-2.

43 Adam DC, Wu P, Wong JY, et al. Clustering and superspreading potential of SARS-CoV-2 infections in Hong Kong. Nat Med 2020;26:1714-9.

$44 \mathrm{Bi} \mathrm{Q}, \mathrm{Wu}$ Y, Mei S, et al. Epidemiology and transmission of COVID-19 in 391 cases and 1286 of their close contacts in Shenzhen, China: a retrospective cohort study. Lancet Infect Dis 2020;20:911-9.

45 Kucharski AJ, Klepac P, Conlan AJK, et al. Effectiveness of isolation, testing, contact tracing, and physical distancing on reducing transmission of SARS-CoV-2 in different settings: a mathematical modelling study. Lancet Infect Dis 2020;20:1151-60.

46 Hellewell J, Abbott S, Gimma A, et al. Feasibility of controlling COVID-19 outbreaks by isolation of cases and contacts. Lancet Glob Health 2020;8:e488-96.

47 Britton T, Ball F, Trapman P. A mathematical model reveals the influence of population heterogeneity on herd immunity to SARSCoV-2. Science 2020;369:846-9.

48 Salathé M, Althaus CL, Neher R, et al. COVID-19 epidemic in Switzerland: on the importance of testing, contact tracing and isolation. Swiss Med Wkly 2020;150:w20225.

49 Wilder-Smith A, Freedman DO. Isolation, quarantine, social distancing and community containment: pivotal role for old-style public health measures in the novel coronavirus (2019-nCoV) outbreak. J Travel Med 2020;27:taaa020.

50 Grzelak L, Temmam S, Planchais C, et al. A comparison of four serological assays for detecting anti-SARS-CoV-2 antibodies in human serum samples from different populations. Sci Transl Med 2020;12:eabc3103.

51 Zhao J, Yuan Q, Wang H, et al. Antibody responses to SARS-CoV-2 in patients with novel coronavirus disease 2019. Clin Infect Dis 2020;71:2027-34.

52 Lassaunière R, Frische A, Harboe ZB. Evaluation of nine commercial SARS-CoV-2 immunoassays. MedRxiv 2020. 